\title{
Emergence of the L phenotype in Group B Streptococci in the South of Ireland
}

\author{
K. HAYES ${ }^{1}$, L. COTTER ${ }^{1}$, L. BARRY ${ }^{2}$ AND F. O'HALLORAN ${ }^{1}$ \\ ${ }^{1}$ Department of Biological Sciences, Cork Institute of Technology, Rossa Avenue, Bishopstown, Cork, Ireland \\ ${ }^{2}$ Department of Microbiology, Cork University Hospital, Wilton, Cork, Ireland
}

Received 1 June 2017; Final revision 27 September 2017; Accepted 9 October 2017;

first published online 6 November 2017

\section{SUMMARY}

Group B Streptococcal isolates $(n=235)$ from the South of Ireland were characterised by serotyping, antimicrobial susceptibility and determination of the phenotypic and genotypic mechanisms of resistance. Resistance to erythromycin and clindamycin was observed in $21 \cdot 3 \%$ and $20.4 \%$ of the total population, respectively. The c-MLSB phenotype was the most common phenotype detected $(62 \%)$, with $\operatorname{ermB}$ being the predominant genetic determinant, present in $84 \%$ of resistant isolates. The rare L phenotype was observed in $2.9 \%(n=7)$ of isolates, four of which harboured the $l s a C$ gene responsible for clindamycin resistance. Serotypes Ia, III and II were the most common amongst the entire study population $(28 \cdot 1 \%, 24 \cdot 7 \%$ and $14 \%$, respectively). Four of the seven L phenotype isolates were serotype III and two of these strains were confirmed as the hypervirulent clone, ST-17 and harboured the $h v g A$ gene. This is the first documented case of the L phenotype in Ireland to date and the study findings emphasise the need for continued monitoring of antibiotic resistance and serotype distribution in GBS isolates from Ireland.

Key words: Microbiology, antibiotic resistance, Streptococcal infections, surveillance.

\section{INTRODUCTION}

Group B Streptococcus (GBS), or Streptococcus agalactiae, frequently colonise the genitourinary tract in pregnant women and can cause invasive disease in neonates, with fatality rates as high as $10 \%$ [1]. Up to $25 \%$ of neonates that survive infection may suffer severe neurological impairment [2]. In recent years, GBS has also emerged as an important pathogen in nonpregnant adults, particularly the elderly and those with underlying diseases such as diabetes [3].

Ten serotypes of GBS exist but studies have shown that serotypes Ia, Ib, II, III and V occur more

\footnotetext{
* Author for correspondence: K. Hayes, Department of Biological Sciences, Cork Institute of Technology, Rossa Avenue, Bishopstown, Cork, Ireland.

(Email: katherine.hayes@mycit.ie)
}

frequently and are largely responsible for invasive disease [1,3]. GBS are further classified by sequence types (ST) based on multilocus sequence typing (MLST), with ST-1, ST-10, ST-17, ST-19 and ST-23 being more frequently associated with invasive infection $[1,4,5]$. The 'hypervirulent' clone ST-17 is often linked with serotype III strains in invasive neonatal disease [6] but recent studies have also shown an association between serotype V, ST-1 strains and invasive disease in non-pregnant adults [7].

Current practice in Ireland for prevention and control of neonatal GBS disease involves a risk-based assessment, as per the Royal College of Obstetricians and Gynaecologists guidelines, with intrapartum antibiotic prophylaxis (IAP) administered only to those considered at significant risk [8]. However, the use of IAP has raised concerns regarding the potential for 
development of antibiotic resistance in GBS isolates [9]. Penicillin remains the first line agent for IAP and treatment of GBS infections [10], however, isolates with reduced susceptibility have been confirmed in some countries [11]. When a patient presents with beta-lactam allergies, alternative treatment options are required, with lincosamides (clindamycin) and macrolides (erythromycin) considered the next choice of treatment, but resistance levels to these antibiotics continue to rise $[3,10]$.

Macrolide resistance is mediated by two mechanisms with ribosomal modification by methylation (encoded by erm genes) being the most common, and efflux pumps. In streptococci, ermB and ermTR genes confer resistance to erythromycin, and constitutive or inducible resistance to clindamycin and streptogramin B antibiotics (c- or i-MLSB phenotypes respectively). Efflux pumps are encoded by mef genes and restrict resistance to 14- and 15-membered macrolides only (e.g. erythromycin), which is associated with the $\mathrm{M}$ phenotype $[12,13]$.

Resistance to clindamycin occurs primarily through erm genes, however, isolates displaying resistance to clindamycin but susceptibility to erythromycin (L phenotype) also occur. This unusual mechanism of lincosamide-specific resistance occurs by enzymatic inactivation of the drug by nucleotidyl-transferases and is mediated by $\ln u$ genes [14]. Furthermore, this type of lincosamide resistance may also occur with cross resistance to streptogramins A and pleuromutilins, designated LSA and LSAP phenotypes, which are mediated by the $\mathrm{ABC}$ transporter, lsaC [15]. A retrospective study conducted at the Centers for Disease Control and Prevention in 2016 confirmed that while the incidence of these rare phenotypes is low, their rates are increasing and thus should be actively monitored [16].

This study aimed to provide current epidemiological data on serotype distribution and antibiotic resistance rates among colonising and invasive GBS isolates in the South of Ireland, and to investigate the genetic basis for antibiotic resistance and the emergence of new strain phenotypes.

\section{MATERIALS AND METHODS}

\section{Bacterial isolates}

A total of 235 GBS isolates collected over a 6-year period (2010-2016) were included in this study. Colonising isolates $(n=201)$ were collected from the
Microbiology Department at Cork University Hospital, obtained from high vaginal swabs (HVS) of women between the ages of 16 and 45 years; one colonising GBS isolate was recovered from a placental swab (PS) and was provided by the National Maternity Hospital, Holles Street, Dublin. Invasive GBS isolates $(n=34)$ were collected from blood culture samples from Cork University Hospital and University Hospital Limerick. Isolates were cultured on Columbia Blood Agar (Fannin Ltd, Dublin, Ireland) and incubated at $37^{\circ} \mathrm{C}$ for $24 \mathrm{~h}$. Isolates were identified as GBS by beta-haemolysis on blood agar, a positive Gram stain and a negative catalase test and identified to species level by MALDI-TOF MS (Bruker Daltonics, Leipzig, Germany).

\section{Antimicrobial susceptibility testing}

Antimicrobial susceptibility of the isolates was assessed by a disk diffusion method, according to EUCAST guidelines (http://www.eucast.org/ast_of_ bacteria/) and all isolates were tested for susceptibility to penicillin, erythromycin, clindamycin, rifampicin and tetracycline. Inducible resistance to clindamycin was determined using the double-disk diffusion method (D test) (EUCAST) and resistance phenotypes were characterised as constitutive (c-MLSB), inducible (i-MLSB), M phenotype (erythromycin resistant, clindamycin sensitive) or L phenotype (erythromycin sensitive, clindamycin resistant). Minimum inhibitory concentration (MIC) testing of erythromycin and clindamycin was performed using MIC Evaluator strips (Oxoid, Basingstoke, UK) and interpreted according to EUCAST breakpoints (http://www.eucast.org/clinical_breakpoints/).

\section{DNA extraction from bacterial cultures}

DNA was extracted using the PureLink Genomic DNA Kit (Invitrogen, ThermoFisher Scientific, California, USA) with minor modifications, which included a mechanical lysis of GBS by bead-beating using the MagNA DNA lyser (Roche, Indiana, USA). Briefly, an overnight culture of GBS in Brain Heat Infusion broth (BHI, Cruinn Diagnostics, Dublin, Ireland) was centrifuged at $6500 \mathrm{rpm}$ for $10 \mathrm{~min}$. The supernatant was removed and the cell pellet was re-suspended in $1 \mathrm{ml}$ TE buffer. To the washed cells, $470 \mathrm{mg}$ of $150-212 \mu \mathrm{m}$ glass beads (Sigma-Aldrich, Missouri, USA) were added and the samples were bead-beated at $6500 \mathrm{rpm}$ for $60 \mathrm{~s}$. Samples were then 
centrifuged at $13000 \mathrm{rpm}$ for $10 \mathrm{~min}$. From each lysed sample, $240 \mu$ l of supernatant was removed and processed using the extraction and purification protocol described by the manufacturer, beginning with the proteinase $\mathrm{K}$ digestion step.

\section{Molecular characterisation of antimicrobial resistance genes}

A multiplex PCR assay was used to detect the presence of the erythromycin resistance genes ermB and $m e f A / E$, with inclusion of an $S$. agalactiae-specific internal control targeting the $c f b$ gene $[17,18]$. Other genes involved in promoting erythromycin resistance (ermTR and ermT) were detected by PCR as previously described $[19,20]$. A multiplex assay was performed to detect the clindamycin resistance genes $l s a C$ and $\ln u B[14,15]$. Additional clindamycin resistance genetic determinants (lnuA, lsaB, lsaE and $v g a C$ ) were investigated by PCR according to previous studies $[21,22]$.

\section{Capsular typing and ST-17 detection}

All isolates were assigned serotypes according to the recommended polymerase chain reaction (PCR)based capsular genotype algorithm [23]. Briefly, isolates were initially serotyped using two multiplex PCR reactions [24] and any serotype VII or nontypeable isolates were further identified by PCR [25]. Detection of the hypervirulent ST-17 clone was performed using a modified version of a multiplex PCR assay targeting a region of the $h v g A$ gene [26].

\section{Statistical analysis}

The $\chi^{2}$ test and Fisher's exact test were used to analyse associations using SPSS v. 24.0 (IBM, UK). A $P$-value $\leqslant 0.05$ was considered statistically significant.

\section{RESULTS}

\section{Antimicrobial susceptibility}

All isolates $(n=235)$ were susceptible to penicillin and rifampicin. Resistance to tetracycline, erythromycin and clindamycin (both constitutive and inducible) was observed in $87 \cdot 2 \%(n=205), 21 \cdot 3 \%(n=50)$ and $20 \cdot 4 \%(n=48)$ of isolates, respectively. Differences in overall resistance rates were evident between invasive $(n=34)$ and colonising $(n=201)$ isolates, with all of the former group being resistant to tetracycline compared with $85 \cdot 1 \%(n=171)$ of colonisers. Similarly, erythromycin resistance was found in $35.3 \%$ (n $=12)$ of invasive and $18.9 \%(n=38)$ of colonising isolates $(P=0.041)$, and clindamycin resistance in $32 \cdot 4 \%$ $(n=11)$ and $18 \cdot 4 \%(n=37)$ of invasive and colonising isolates, respectively $(P=0 \cdot 069)$.

\section{Macrolide resistance phenotypes and genotypes}

In total, 50 isolates were resistant to erythromycin ( $n=12$ invasive; $n=38$ colonising) and of these, $62 \%$ $(n=31)$ exhibited the c-MLSB phenotype $(n=9$ invasive; $n=22$ colonising), while $20 \%(n=10)$ were of the i-MLSB phenotype ( $n=2$ invasive; $n=8$ colonising) and $18 \%(n=9)$ belonged to the M phenotype $(n=1$ invasive, $n=8$ colonising) (Table 1). The erm $B$ gene was the most frequent $(84 \%, n=42)$ among macrolide resistant isolates while $m e f A / E$ and $e r m T R$ genes were each detected in 18\% $(n=9)$ of isolates; ermT was restricted to a single isolate, which was also positive for the $\operatorname{erm} B$ gene. One isolate harboured a combination of ermB and mefAlE genes, and another was positive for both ermTR and mefA/E. All 31 isolates exhibiting the c-MLSB phenotype were ermB positive and three of these $(9 \cdot 7 \%)$ also harboured the ermTR gene. The erm $\mathrm{B}$ gene was detected in all 10 isolates displaying the i-MLSB phenotype and five of these isolates were positive for the ermTR gene; another single isolate harboured the ermT gene. All M phenotype $(n=9)$ representatives contained the $m e f A / E$ resistance determinant, with only one isolate being additionally positive for ermB (Table 1).

\section{Characterisaton of the $L$ phenotype}

The L phenotype of erythromycin sensitivity and clindamycin resistance was identified in 2.9\% $(n=7)$ of all GBS isolates and confirmed by MIC susceptibility testing (Table 2). Four isolates were positive for the the $l s a C$ gene and the remaining three were negative for all genetic deteminants investigated. The L phenotype was not detected among invasive isolates.

\section{Serotype distribution amongst GBS populations}

Nine serotypes were identified among the total study collection; serotype IX was not found and one isolate was non-typeable by both PCR methods. The most common serotypes were Ia $(28 \cdot 1 \%)$, III $(24 \cdot 7 \%)$, II $(14 \%), \mathrm{V}(12 \cdot 8 \%)$ and Ib (11.9\%), and serotypes IV, VI, VII and VIII accounted for $4 \cdot 3 \%, 1 \cdot 3 \%, 1 \cdot 7 \%$, 
Table 1. Genotypic and phenotypic characterisation of group B streptococcal isolates resistant to erythromycin

\begin{tabular}{|c|c|c|c|c|c|c|}
\hline \multirow[b]{4}{*}{ Genotype } & \multicolumn{6}{|c|}{ Phenotype } \\
\hline & \multicolumn{3}{|c|}{ Invasive $(n=12)$} & \multicolumn{3}{|c|}{ Colonising $(n=38)$} \\
\hline & \multicolumn{6}{|c|}{ Macrolide-resistant } \\
\hline & $\begin{array}{l}\text { c-MLSB } \\
(n=9)\end{array}$ & $\begin{array}{l}\text { i-MLSB } \\
(n=2)\end{array}$ & $\begin{array}{l}M \\
(n=1)\end{array}$ & $\begin{array}{l}\text { c-MLSB } \\
(n=22)\end{array}$ & $\begin{array}{l}\text { i-MLSB } \\
(n=8)\end{array}$ & $\begin{array}{l}\text { M } \\
(n=8)\end{array}$ \\
\hline erm $B+$, mef $A / E-$, ermTR-, ermT- & 8 & 1 & & 20 & 3 & \\
\hline ermB+, mefAlE-, ermTR+, ermT- & 1 & 1 & & 2 & 4 & \\
\hline ermB+, mefA/E-, ermTR-, ermT+ & & & & & 1 & \\
\hline ermB+, mef $A / E+$, ermTR-, ermT- & & & & & & 1 \\
\hline ermB-, mef $A / E+$, ermTR-, ermT- & & & 1 & & & 6 \\
\hline ermB-, mef $A / E+$, ermTR+, erm $T-$ & & & & & & 1 \\
\hline
\end{tabular}

c-MLSB, constitutive macrolide and lincosamide resistance; i-MLSB, inducible clindamycin resistance in the presence of erythromycin; $\mathrm{M}$, resistance to erythromycin only.

Table 2. Characterisation of $L$ phenotype isolates of group B streptococci

\begin{tabular}{|c|c|c|c|c|}
\hline \multirow[b]{2}{*}{ Isolate no. } & \multicolumn{2}{|l|}{$\operatorname{MIC}(\mu \mathrm{g} / \mathrm{ml})$} & \multirow[b]{2}{*}{ Genetic determinant } & \multirow[b]{2}{*}{ Serotype } \\
\hline & Clindamycin & Erythromycin & & \\
\hline 1 & $\geqslant 256$ & $0 \cdot 25$ & - & $\mathrm{V}$ \\
\hline 2 & $\geqslant 256$ & $0 \cdot 25$ & - & $\mathrm{V}$ \\
\hline 3 & $\geqslant 256$ & $0 \cdot 5$ & - & II \\
\hline 4 & 2 & 0.03 & lsaC & III \\
\hline 5 & 1 & 0.03 & lsaC & $\mathrm{III}^{\mathrm{a}}$ \\
\hline 6 & 1 & 0.03 & lsaC & III \\
\hline 7 & 1 & 0.03 & lsaC & III $^{\mathrm{a}}$ \\
\hline
\end{tabular}

All isolates were screened for the presence of $\ln u A$, $\ln u B$, lsaB, $l s a C$, ls $a E$ and $\operatorname{vg} a C$ determinants. Isolates 1-6 were of high vaginal swab origin, isolate 7 was from a placental swab.

${ }^{\mathrm{a}}$ Isolates of the ST-17 clone.

and $0.85 \%$ of isolates, respectively. Among colonising isolates the predominant serotypes were serotypes Ia $(29 \cdot 4 \%)$, III $(25.9 \%)$, II $(15 \cdot 4 \%), \mathrm{V}(11.4 \%)$ and Ib $(9 \cdot 95 \%)$. However, the serotype distribution for invasive isolates differed, with $\mathrm{Ib}(23 \cdot 5 \%)$, Ia $(20 \cdot 6 \%)$, V $(20 \cdot 6 \%)$ and III $(17 \cdot 6 \%)$ being the most prevalent, but the difference between the two isolate groups did not reach statistical significance $(P=0 \cdot 081)$.

The hyper-virulent clone, ST-17, was identified in $12 \cdot 3 \%(n=29)$ of all isolates, with $20 \cdot 6 \%(n=7)$ and $10.9 \%(n=22)$ accounting for invasive and colonising populations. All, but one, of the ST-17 isolates were serotype III $(P<0.0005)$, the other isolate being confirmed by both PCR methods as serotype IV.

Table 3 shows that there was no significant difference in serotype distribution between neonatal and adult invasive isolates $(P=0 \cdot 378)$. Two invasive adult isolates were serotype III and ST-17, and another strain of serotype IV/ST-17 was also confirmed from an adult sample. Of the 52 serotype III colonising isolates, $42 \cdot 3 \%(n=22)$ were ST- 17 .

Within the 50 isolates resistant to erythromycin, serotype III predominated $(n=15)$ followed by serotype $\mathrm{V}(n=12)$, Ia $(n=8)$, II $(n=6)$ and $\mathrm{Ib}(n=4)$. Both serotypes IV and VII were found in two isolates each and serotype VI was identified in a single isolate only. The majority of the 15 serotype III erythromycin resistant isolates expressed the c-MLSB phenotype $(86.7 \%)$ and all harboured the ermB gene; the c-MLSB phenotype was also found in 9 of 12 serotype $\mathrm{V}$ isolates but these harboured varied erythromycin resistance determinants (Table 4). Significant associations were found between serotype Ia and the $\mathrm{M}$ phenotype $(P=0.0001)$ and serotype Ia and the 
Table 3. Correlation of sample origin and serotype distribution amongst invasive and colonising isolates of group $B$ streptococci

\begin{tabular}{|c|c|c|c|c|c|c|}
\hline \multirow[b]{2}{*}{ Serotype } & \multicolumn{2}{|c|}{ Invasive } & \multirow[b]{2}{*}{$\begin{array}{l}\text { Unknown }^{\mathrm{a}} \\
(n=7)\end{array}$} & \multicolumn{2}{|c|}{ Colonising } & \multirow{2}{*}{$\begin{array}{l}\text { Total } \\
(n=235)\end{array}$} \\
\hline & $\begin{array}{l}\text { Neonatal } \\
(n=7)\end{array}$ & $\begin{array}{l}\text { Adult } \\
(n=20)\end{array}$ & & $\begin{array}{l}\text { HVS }^{\mathrm{b}} \\
(n=200)\end{array}$ & $\begin{array}{l}\mathrm{PS}^{\mathrm{c}} \\
(n=1)\end{array}$ & \\
\hline Ia & 1 & 4 & 2 & 59 & & 66 \\
\hline $\mathrm{Ib}$ & & 6 & 2 & 20 & & 28 \\
\hline II & 1 & 1 & & 31 & & 33 \\
\hline III & $3^{\mathrm{d} 100}$ & $2^{\mathrm{d} 100}$ & $1^{\mathrm{d} 100}$ & $51^{\mathrm{d} 41 \cdot 2}$ & $1^{\mathrm{d} 100}$ & $58^{\mathrm{d} 48 \cdot 3}$ \\
\hline IV & & $2^{\mathrm{d} 50}$ & & 8 & & $10^{\mathrm{d} 10}$ \\
\hline V & 1 & 5 & 1 & 23 & & 30 \\
\hline VI & 1 & & & 2 & & 3 \\
\hline VII & & & & 4 & & 4 \\
\hline VIII & & & 1 & 1 & & 2 \\
\hline IX & & & & & & \\
\hline NT & & & & 1 & & 1 \\
\hline
\end{tabular}

a Lack of clinical information.

${ }^{\mathrm{b}}$ High vaginal swab.

${ }^{\mathrm{c}}$ Placental swab.

${ }^{\mathrm{d}}$ Percentage of ST-17 isolates within group.

Table 4. Correlation between serotype, phenotype and genotype of group B streptococcal isolates

\begin{tabular}{|c|c|c|c|c|}
\hline CPS Serotype (No. of isolates) & E-resistant $^{\mathrm{a}}$ & DA-resistant ${ }^{b}$ & Phenotype & Genotype \\
\hline Ia (8) & 8 & & $\begin{array}{l}\text { c-MLSB (3) } \\
\text { M (5) }\end{array}$ & $\begin{array}{l}\operatorname{ermB}(3), \operatorname{ermTR}(1) \\
m e f A / E(5), \operatorname{erm} B(1)\end{array}$ \\
\hline $\mathrm{Ib}(4)$ & 4 & & $\begin{array}{l}\text { c-MLSB (2) } \\
\text { i-MLSB (2) }\end{array}$ & $\begin{array}{l}\operatorname{ermB}(2) \\
\operatorname{ermB}(2), \operatorname{ermTR}(1)\end{array}$ \\
\hline II (7) & 6 & 1 & $\begin{array}{l}\text { c-MLSB (3) } \\
\text { i-MLSB (3) } \\
\text { L (1) }\end{array}$ & $\begin{array}{l}\operatorname{ermB}(3) \\
\operatorname{erm} B(2), \operatorname{ermTR}(2), \operatorname{ermT}(1)\end{array}$ \\
\hline III (19) & 15 & 4 & $\begin{array}{l}\text { c-MLSB (13) } \\
\text { i-MLSB (2) } \\
\text { L (4) }\end{array}$ & $\begin{array}{l}\operatorname{erm} B(13) \\
\operatorname{erm} B(2) \\
\text { lsaC (4) }\end{array}$ \\
\hline IV (2) & 2 & & $\begin{array}{l}\text { c-MLSB (1) } \\
\text { i-MLSB (1) }\end{array}$ & $\begin{array}{l}\operatorname{erm} B(1) \\
\operatorname{erm} B(1), \operatorname{ermTR}(1)\end{array}$ \\
\hline V (14) & 12 & 2 & $\begin{array}{l}\text { c-MLSB (8) } \\
\text { i-MLSB (2) } \\
\text { M (2) } \\
\text { L (2) }\end{array}$ & $\begin{array}{l}\operatorname{ermB}(8), \operatorname{ermTR}(1) \\
\operatorname{erm} B(2), \operatorname{ermTR}(2) \\
m e f A / E(2)\end{array}$ \\
\hline VI (1) & 1 & & c-MLSB (1) & $\operatorname{erm} B(1)$ \\
\hline VII (2) & 2 & & M (2) & $m e f A / E(2), \operatorname{ermTR}(1)$ \\
\hline
\end{tabular}

${ }^{\mathrm{a}}$ E-resistant $=$ all erythromycin-resistant isolates resistant or sensitive to clindamycin.

${ }^{\mathrm{b}}$ DA-resistant $=$ isolates resistant to clindamycin but susceptible to erythromycin .

mefA/E gene $(P=0 \cdot 00046)$. Serotype $\mathrm{V}$ was also frequent among the invasive erythromycin-resistant population $(41 \cdot 7 \%)$, while serotype III predominated among colonising isolates resistant to erythromycin $(31.6 \%)$ but there was no significant difference in serotype distribution within the two populations $(P=0 \cdot 317)$. Three of the 12 erythromycin resistant invasive isolates were ST-17 and were also resistant to clindamycin; correspondingly, three colonising isolates of the resistance phenotype were members of the same ST-17 clone.

Four of the seven isolates exhibiting the $\mathrm{L}$ phenotype were serotype III, two were of serotype V and one was serotype II (Table 2). The four L phenotype 
serotype III isolates harboured the $l s a C$ gene and two of these were ST-17.

\section{DISCUSSION}

Despite recent reports of reduced beta-lactam susceptibility among GBS isolates [11], data from this study indicate that susceptibility to penicillin is universal amongst GBS strain populations in Ireland. This reaffirms the appropriateness of penicillin as the antibiotic of choice for first line IAP and for the treatment of GBS infections. The incidence of erythromycin resistance found $(21 \cdot 3 \%)$ is similar to previous studies conducted in Ireland $(18.6 \%)$, Poland $(18.4 \%)$ and Italy $(20 \%)[5,27,28]$, but lower than rates in France $(34 \cdot 7 \%)$ and Taiwan (46\%) [29, 30]. Interestingly the rate of clindamycin resistance in the current study indicates that resistance has increased in the Irish population from $15 \cdot 25 \%$ [5] to $20 \cdot 4 \%$ and is higher than reports from other countries, including Poland and Africa [27, 31]. As with other countries, a high rate of tetracycline resistance $(87 \cdot 2 \%)$ was evident for the isolates reported here $[28,29]$.

Colonising GBS isolates can be reservoirs of virulence and antibiotic resistance markers and vaginal colonisation may pose a risk for subsequent invasive disease in neonates. Both erythromycin and clindamycin resistance are higher among invasive $(35.3 \%$ and $32.4 \%$ respectively) than colonising isolates $(18.9 \%$ and $18.4 \%$ respectively), although this only reached statistical significance for erythromycin resistance $(P=0 \cdot 041)$.

Regarding antibiotic resistance mechanisms, the c-MLSB phenotype predominated overall (62\%) and was more frequent among invasive $(75 \%)$ than colonising isolates $(57.9 \%)$. The ermB gene was the most common resistance determinant identified (84\%) and this finding corroborates the results of the most recent Irish study [5]. The presence of the ermT gene, although only in a single isolate, is notable as it was associated with the i-MLSB phenotype. This determinant may be of importance as it is plasmid-borne and has been shown to be readily transferable between GBS and Enterococcus faecalis, which could promote its wider dissemination [20]. It is interesting to note the co-occurrence of ermB and ermTR genes in eight isolates and $m e f A / E$ and $e r m T R$ genes in another isolate. Co-occurrence of ermB and ermTR has also been noted in other studies, although in a lower number of isolates $[28,29,32,33]$.
The L phenotype was first documented in E. faecium in 1999 [14] and followed shortly after by the first report in a GBS strain in Canada in 2001 [19]. Since then there have been reports of the L phenotype in Spain [21], Korea [32], the USA [34], Argentina [35] and South Africa [36] with incidence rates varying from $0 \cdot 26 \%$ in Italy [28] to $15.9 \%$ in Korea [32]. To date, the phenotype had not been confirmed in GBS isolates from Ireland but was found here to represent almost 3\% of isolates which, interestingly, were collected in the latter part of the study period (2015-2016).

Four of the seven L phenotype isolates harboured the $l s a C$ gene and were serotype III. This observation concurs with reports from previous studies $[15,16,21$, 32, 37]. Moreover, the three other isolates of this phenotype with an MIC for clindamycin $>256 \mu \mathrm{g} / \mathrm{ml}$ were negative for all other determinants tested including the $l n u, l s a B, l s a E$ and $v g a C$ genes. Two of these isolates were confirmed as serotype $\mathrm{V}$ and the other was serotype II. To the best of our knowledge, this is the first documented report of GBS isolates with the $\mathrm{L}$ phenotype and characterised as containing the $l s a C$ gene in Ireland. Although the L phenotype was exclusively identified in colonising isolates in this study, it has been found by others in patients with invasive GBS disease [16, 37]. Also of interest, two of the seven isolates were confirmed as belonging to the hypervirulent ST-17 clone. The emergence of this uncommon phenotype in Irish GBS strains corroborates the need for continued surveillance of emerging antibiotic resistance, as recently reported in the USA [16]. This USA study also revealed the presence of L phenotypes with cross-resistance to streptogramins A and pleuromutilins, the LSA/LSAP phenotypes, which have been reported elsewhere [15, 37]. Another recent study using whole genome sequencing to identify resistance mechanisms in GBS, identified the LSA/LSAP phenotypes using this approach and highlighted the co-occurrence of certain genes, particularly $l s a C$ and ermB, amongst isolates displaying this phenotype [33]. While we did not specifically investigate the LSA/LSAP phenotypes, they may be clinically significant going forward and support the need for further study into the development of alternative antimicrobials.

Here, nine serotypes were confirmed among the isolate collection and only one isolate proved nontypeable by both PCR methods used. Overall, serotype Ia predominated $(28 \cdot 1 \%)$, followed by types III, II and $\mathrm{V}$. This distribution is comparable with a previous Irish study [5] and indicates a level of stability within the GBS 
population in this country. Serotype III was also predominant among erythromycin-resistant isolates in contrast to a previous study in the South of Ireland where serotype $\mathrm{V}$ was more frequently associated with erythromycin-resistant strains [38].

The ST-17 lineage accounted for $12 \cdot 3 \%$ of all isolates studied with rates of $20 \cdot 6 \%$ and $10.9 \%$ in the invasive and colonising populations respectively. The high prevalence of this clone in the latter group is of concern because of its potential to progress to invasive disease, thus highlighting the importance of monitoring reservoirs of colonising strains. Three of the ST-17 invasive isolates were of neonatal origin and of serotype III, and three were from invasive adult infections, but of different serotypes (III and IV) which may suggest the occurrence of capsular antigen switching as reported in other studies [5, 39]. Together, these findings serve to emphasise the need for continuous surveillance of serotypes which is highly relevant to the development of vaccines [40].

In conclusion, the increase in resistance to erythromycin and clindamycin in GBS strains is of significance particularly for individuals with penicillin allergies. Emergence of the L phenotype in the Irish GBS population is a concern and warrants continued surveillance of resistance rates and further elucidation of the underlying genetic mechanisms.

\section{ACKNOWLEDGEMENTS}

The authors are extremely grateful to staff at the Microbiology Department of University Hospital Limerick and Cork University Hospital for the provision of GBS isolates. The authors also thank the Microbiology Department of The National Maternity Hospital, Holles Street. This work was supported by the RÍSAM Scholarship Programme of Cork Institute of Technology.

\section{DECLARATION OF INTEREST}

The authors declare no conflict of interest.

\section{REFERENCES}

1. Edmond KM, et al. Group B streptococcal disease in infants aged younger than 3 months: systematic review and meta-analysis. Lancet 2012; 379: 547-556.

2. Libster R, et al. Long-term outcomes of group B streptococcal meningitis. Pediatrics 2012; 130: e8-e15.

3. Lamagni TL, et al. Emerging trends in the epidemiology of invasive group B streptococcal disease in England and Wales, 1991-2010. Clinical Infectious Diseases 2013; 57: 682-688.

4. Jones N, et al. Multilocus sequence typing system for group B streptococcus. Journal of Clinical Microbiology 2003; 41: 2530-2536.

5. Meehan M, Cunney R, Cafferkey M. Molecular epidemiology of group B streptococci in Ireland reveals a diverse population with evidence of capsular switching. European Journal of Clinical Microbiology \& Infectious Diseases 2014; 33: 1155-1162.

6. Tazi A, et al. The surface protein HvgA mediates group B streptococcus hypervirulence and meningeal tropism in neonates. Journal of Experimental Medicine 2010; 207: 2313-2322.

7. Flores AR, et al. Sequence type 1 group B Streptococcus, an emerging cause of invasive disease in adults, evolves by small genetic changes. Proceedings of the National Academy of Sciences, USA 2015; 112: 6431-6436.

8. Royal College of Obstetricians and Gynaecologists. The Prevention of Early-Onset Neonatal Group B Streptococcal Disease. Green Top Guideline No. 36. London: RCOG, 2012.

9. Phares CR, et al. Epidemiology of invasive group B streptococcal disease in the United States, 1999-2005. Journal of American Medical Association 2008; 299: 2056-2065.

10. Verani JR, McGee L, Schrag SJ. Prevention of perinatal group B streptococcal disease - revised guidelines from CDC. Morbidity and Mortality Weekly Report 2010; 59(RR-10): 1-36.

11. Kimura K, et al. First molecular characterization of group B streptococci with reduced penicillin susceptibility. Antimicrobial Agents and Chemotherapy 2008; 52: 2890-2897.

12. Roberts MC, et al. Nomenclature for macrolide and macrolide-lincosamide-streptogramin B resistance determinants. Antimicrobial Agents and Chemotherapy 1999; 43: 2823-2830.

13. Leclercq R. Mechanisms of resistance to macrolides and lincosamides: nature of the resistance elements and their clinical implications. Clinical Infectious Diseases 2002; 34: 482-492.

14. Bozdogan B, et al. A new resistance gene, $\operatorname{lin} B$, conferring resistance to lincosamides by nucleotidylation in Enterococcus faecium HM1025. Antimicrobial Agents and Chemotherapy 1999; 43: 925-929.

15. Malbruny B, et al. Cross-resistance to lincosamides, streptogramins A, and pleuromutilins due to the $l s a(C)$ gene in Streptococcus agalactiae UCN70. Antimicrobial Agents and Chemotherapy 2011; 55: 1470-1474.

16. Hawkins PA, et al. Cross-resistance to lincosamides, streptogramins A and pleuromutilins in Streptococcus agalactiae isolates from the USA. Journal of Antimicrobial Chemotherapy 2017; 72: 1886-1892.

17. Sutcliffe J, et al. Detection of erythromycin-resistant determinants by PCR. Antimicrobial Agents and Chemotherapy 1996; 40: 2562-2566.

18. Ke D, et al. Development of conventional and real-time PCR assays for the rapid detection of group B streptococci. Clinical Chemistry 2000; 46: 324-331. 
19. De Azavedo JC, et al. Prevalence and mechanisms of macrolide resistance in invasive and non-invasive group B streptococcus isolates from Ontario, Canada. Antimicrobial Agents and Chemotherapy 2001; 45: 3504-3508.

20. Compain F, et al. Molecular characterization of Streptococcus agalactiae isolates harboring small erm (T)-carrying plasmids. Antimicrobial Agents and Chemotherapy 2014; 58: 6928-6930.

21. Arana DM, et al. First clinical isolate in Europe of clindamycin-resistant group B Streptococcus mediated by the $\operatorname{lnu}(\mathrm{B})$ gene. Revista Española de Quimioterapia 2014; 27: 106-109.

22. Douarre PE, et al. Host specificity in the diversity and transfer of lsa resistance genes in group B Streptococcus. Journal of Antimicrobial Chemotherapy 2015; 70: 3205-3213.

23. Yao K, et al. Capsular gene typing of Streptococcus agalactiae compared to serotyping by latex agglutination. Journal of Clinical Microbiology 2013; 51: 503-507.

24. Poyart C, et al. Multiplex PCR assay for rapid and accurate capsular typing of group B streptococci. Journal of Clinical Microbiology 2007; 45: 1985-1988.

25. Imperi M, et al. A multiplex PCR assay for the direct identification of the capsular type (Ia to IX) of Streptococcus agalactiae. Journal of Microbiological Methods 2010; 80: 212-214.

26. Gosiewski T, Brzychczy-Włoch M, Heczko PB. The application of multiplex PCR to detect seven different DNA targets in group B streptococci. Folia Microbiologica (Praha) 2012; 57: 163-167.

27. Sadowy E, Matynia B, Hryniewicz W. Population structure, virulence factors and resistance determinants of invasive, non-invasive and colonizing Streptococcus agalactiae in Poland. Journal of Antimicrobial Chemotherapy 2010; 65: 1907-1914.

28. Bezares B, et al. Streptococcus agalactiae from pregnant women: antibiotic and heavy-metal resistance mechanisms and molecular typing. Epidemiology and Infection 2016; 144: 3205-3214.

29. Bergal A, et al. Molecular epidemiology and distribution of serotypes, genotypes, and antibiotic resistance genes of Streptococcus agalactiae clinical isolates from Guelma, Algeria and Marseille, France. European Journal of Clinical Microbiology and Infectious Diseases 2015; 34: 2339-2348.
30. Hsueh PR, et al. High incidence of erythromycin resistance among clinical isolates of Streptococcus agalactiae in Taiwan. Antimicrobial Agents and Chemotherapy 2001; 45: 3205-3208.

31. Belard S, et al. Streptococcus agalactiae serotype distribution and antimicrobial susceptibility in pregnant women in Gabon, Central Africa. Scientific Reports 2015; 5: 17281

32. Seo YS, et al. Changing molecular epidemiology of group B streptococcus in Korea. Journal of Korean Medical Science 2010; 25: 817-823.

33. Metcalf BJ, et al. Short-read whole genome sequencing for determination of antimicrobial resistance mechanisms and capsular serotypes of current invasive Streptococcus agalactiae recovered in the USA. Clinical Microbiology and Infection 2017; 23: 574.e7-574.e14.

34. Gygax SE, et al. Erythromycin and clindamycin resistance in group B streptococcal clinical isolates. Antimicrobial Agents and Chemotherapy 2006; 50: 1875-1877.

35. Faccone D, et al. Multiple clones of Streptococcus agalactiae harbouring $\operatorname{lnuB}$ gene. Journal of Infection in Developing Countries 2010; 4: 580-582.

36. Bolukaoto JY, et al. Antibiotic resistance of Streptococcus agalactiae isolated from pregnant women in Garankuwa, South Africa. BMC Research Notes 2015; 8: 364 .

37. Björnsdóttir ES, et al. Changing epidemiology of group B streptococcal infections among adults in Iceland: 1975-2014. Clinical Microbiology and Infection 2016; 22: 379.e9-379.e16.

38. Kiely RA, Lucey B, Cotter L. Analysis of phenotype, genotype and serotype distribution in erythromycinresistant group B streptococci isolated from vaginal flora in Southern Ireland. Epidemiology and Infection 2010; 138: 286-291.

39. Bellais $\mathbf{S}$, et al. Capsular switching in group B Streptococcus CC17 hypervirulent clone: a future challenge for polysaccharide vaccine development. Journal of Infectious Diseases 2012; 206: 1745-1752.

40. Leroux-Roels G, et al. A randomized, observer-blind Phase Ib study to identify formulations and vaccine schedules of a trivalent Group B Streptococcus vaccine for use in non-pregnant and pregnant women. Vaccine 2016; 34: 1786-1791. 